The first case to occur here was that of an Arab who had just returned from a pilgrimage to Mecca. The villages on the road through which he passed weresubsequently attacked by the disease. Whether his advent had any relation to the present outbreak I am unable to say, but it certainly looked very suspicious.

Tne present epidemic has been no respecter of persons, rich and poor, young and old, males and females, being alike attacked, nor has its distribution been confined to any particular area of those towns visited. In the towns attacked, the disease remained active for from three to four weeks, then suddenly disappeared.

Incubation Period.

One of my servants, a boy 12 years of age, ran away and slept in an infected compound, where there were 2 cases at the time. He developed the disease on the third day, and died on the fifth day. The Residency where the boy was living is two miles away from the nearest infected area, and at that time the strictest isolation was being enforced, so that as far as I know he came into contact with no infected cases. This case would show that the incubation period was as short as three days.

\section{Food as a Source of Infection.}

One case, a child 3 months old, developed the disease, and died; it had not received any other kind of food, its mother was healthy at the time, and did not subsequently contract the disease, so that I think in this particular case food did not act as a carrier of the disease.

The disease is, I think, not acutely infective; I myself went freely amongst then. I knew of one case where a man slept in a house in which a patient had died seven days before ; this man afterwards contracted the disease, he not having visited an infected area after having slept in the infected house.

\section{Prodromata.}

None were observed in the rapidly fatal cases, but in the milder ones, epistaxis, accompanied by a violent headache and a feeling of lassitude, was noticed.

\section{Symptoms.}

These were typical, with the exception of the eruption, which was rarely noticed in adults, probably owing to the difficulty in detecting it in dark-skinned races; in the case of children it evidenced itself in the form of an herpetical eruption on the backs of the hands, wrists, and fingers, back of the neck, dorsum of feet, and over the clavicles. The tongue was dry and covered with a thick white far ; constipation was the rule.

There was nothing characteristic about the fever, no definite course being followed. In the malignant cases the temperature was sometimes subnormal.

Retraction of the head and neck was present in all cases.

Kernig's sign was present in 90 per cent. of the cases examined.

$$
\text { Complications. }
$$

Pneumonia, bronchitis, and arthritis were observed.

$$
\text { Sequelae. }
$$

Blindness, deafness, and partial paralysis.

\section{Prognosis.}

The prognosis was favourable in those who survived the first four or five days; the fatal cases as a rule died in from one to three days.

\section{Treatment.}

Pargatives and opiates were administered with doubtful result.

\section{Mortality.}

Three to 5 per cent. of the total population of infected towns were attacked, with a death-rate of 50 per cent. of the infected cases.

Rapidity of onset was a marked feature of the outbreak. To cite a case : Two men went to draw water from the river, about 300 yards off; both these men were rendered unconscious, and had to be carriej back, both eventually dying.

Malignant cases occurred as a rule during the first week of outbreak in a town, and the milder ones towards its close:

\section{Isolation}

Strict isolation proved effectual here at the Residency, where there is a population of about 250 inhabitants, no cases having occurred, although the epidem!c was raging all around within a radius of two to three miles.

\section{Pathology.}

Lumbar puncture revealed a cloudy fluid somewhat flocculent, and occasionally blood-stained.

Nothing was observed on blood examination. The following are the notes of the post-mortem examination in the case of a man 30 years of age : Duration of disease five days; delirium set in on the first day, and persisted till the end. The membranes at the base of the brain were deeply congested, and fibrinous deposits and some purulent points were discovered beneath the dura mata in the region of the pons and cerebellum; in parts the membranes were adherent to the cortex of the brain. The spinal membranes were similarly hyperaemic. and pus was present in parts beneath the membranes. All the other organs were apparently healthy.

What struck me as a very strange coincident was the simultaneous appearance of spotted fever in New York, Europe, and Northern Nigeria. Was the sun spot which was visible at that time a predisposing factor in its production?

\section{TREATMENT OF PUERPERAL ECLAMPSIA.}

BY ARTHUR C. J. WILSON, L.R.C.P.EDIN., M.R.C.S.ENG., Penistone, Yorks.

I HAVE read with great interest the report of the discussion on eclampsia occurring in pregnancy at the last meeting of the British Medical Association, and also the leading article on the subject in the British Medical Journal of September 23rd.

There is one method of treatment that I do not see definite]y mentioned, though I have carried it out during the last twentynine years. It has much to recommend it in country practice, where, owing to the distance a patient may live away from the medical attendant, frequent visiting is impossible.

This treatment is, when called to a case of eclampsia, to commence at once the administration of chloroform, to give it slowly till the patient is fully under its influence; to keep her under till labour is terminated, so that she shall be partially under its influence for some time afterwards. This treatment I have found very successiul, and have never seen any recurrence of the convulsions after labour, provided a short time only has elapsed after the first convulsion before the administration of chloroform is commenced.

The following cases illustrate this treatment :

Case r.

Called to a multipara at 2 a.m. Third pregnancy. Found her insensible, the convulsions being almost tetanio in character. At once gave chloroform till the patient was fully under its influence, with complete muscular relaxation. Made a vaginal examination, and found the os slightly dilated with breech presentation. Introduced Barnes's bags and terminated labour in about an hour, giving chloroform slowly all the time. The patient slept eight hours afterwards. When she regained consciousness she said she felt well, except feeling sore all over from the effect of the convulsions. The child was alive.

CASE II.

Primipara, unmarried, 20 years of age. She had been treated by an unqualifed man for "ovarian dropsy" "Her parents did not know she was pregnant. I found her lying insensible, having had a serere convulsion. Another commenced soon after my arrival, so I at once administered chloroform till there was complete muscular relaxation. I then easily dilated the os with my hand and terminated labour with the forceps. Patient slept several hours and felt well on regaining consoiousness. There was no return of the convulsions. The child was living.

Primipara, under the care of another practitioner. He administered cbloroform and continued it at intervals for some hours; buit as he was taken ill he asked me to attend the case. I saw the case trrelre hours after the first convulsion, and as the os was fairly dilated I gave more chloroform and terminated the labour with the forceps. She had no more convulsions and made a good recovery. The child was alive.

CASE IV.

Multipara, about 36 years of age; very stout. Only five months advanced in pregnancy. Convulsions very severe on my arrival. Gave chloroform fully. Sent for my assistant and cleared out the uterus. Patient slept some hours and had no more convulsions.

Case v.

Primipara ; short and very stout. Strongly convulsed on my arrival. Gave chloroform fully. Sent for my assistant. Dilated the os with my hand and terminated labour with the forceps. Pdtient slept some 
hoturs. There was no return of the convulsions, and she made a good recovery.

In the following four cases chloroform was not administered at once, other treatment being tried first:

Case vr.

Multipara. Saw the case in the morning; convulsions had ceased, but the patient lay in a semi-conscious state. Full doses of chloral prescribed with instructions that I was to be sent for if the patient became worse. Through negligence I was not sent for till next morning, when I found the patient quite insensible with stertorous breathing, the lower jaw dropped, the pulse very rapid and sosrcely perceptible at the wrist. I thought the case was hopeless, but not liking to let her die undelivered I carefully administered a little chloroform. The breathing became more natural and the pulse became slower and stronger, so I sent for my aesistant and while he administered chloroform I dilated the os and delivered with the forceps. The chlld was dead. The patient slept quietly afterwards, but as the pulse became very weak I tried to give a little nourishment. This was immediately followed by another convulsion, so I at once administered chloroform again. She slept for some hours and there was no return of the convulsions. Bhe made a good recovery.

CASE vir.

Primipara. Attended her for albuminuria and dropsy two days before convulsions occurred. Ordered saline purge and to lie in bed. When the convulsions commenced full doses of chloral were given. After fortyeight hours her condition was getting worse. The os was partially dilated, so I administered chloroform and applied the forceps. She never thoroughly regained consciousness, and died twelve hours afterwards. The ohild was dead.

Case VIII.

Primipara. Convulsions commenced and case attended by my assistant for two days, chloral and hyoscyamus being administered. He then asked me to see the case. I gave chloroform and terminated labour with the forceps. No convulsions afterwards, but not thoroughly conscious for some time. She died at the end of the week with apoplectic symptoms.

Cast ix.

Multipara. My assistant was called to the case at I a.m. I was sent for at 9 a.m., and found that she had had convulsions all night. Gave chloroform and terminated labour with the forceps. Child alive. Convulsions commenced again directly the effect of the chloroform went off, so my son, who had assisted me, stayed with the patient about four hours, administering chloroform on the slightest return of the convulsions. She was partially insensible for two days and then made a good recovery. Hypodermic injections of morphine were given in this case.

These are not all the cases I have attended, but they are all I can call to mind at the present time, but in no single case in which I commenced the administration soon after the convulsions have commenced has there been any return of the convulsions after delivery.

It takes a good "pluck" sometimes to put an insensible woman fully under chloroform, but the effect is marvellous when done.

If the chloroform is administered slowly, and plenty of time allowed so as to get complete muscular relaxation, I have never had any difficulty in dilating the os with the hand, and it can be done without any laceration if due care is taken. I have also had no difficulty with post partum haemorrhage, as the uterus can be well syringed out with hot antiseptic solution and the uterus controlled with the hand while the patient is well under chloroform.

Knowing the good results obtained by the administration of chloroform in uraemic convulsions, has made me rely upon it in puerperal convulsions. A few weeks since I had a case of uraemic convulsions in a child seven weeks old, following scarlet fever. No urine was passed for thirty.six hours. I administered chloroform five times till complete muscular relaxation occurred. The child slept some hours after each administration, and made a good recovery. The points I wish to emphasize are these :

First, that in cases of eclampsia occurring in pregnancy where chloroform was given fully soon after the first convulsion, and when its effect was kept up some time after the termination of labour, no more convulsions occurred.

Secondly, that terminating labour by dilatation of the os can be, as a rule, easily carried out, provided the patient is kept fully under chloroform, and seems to have no effect in causing a return of the convulsions.

Thiraly, in nearly all cases where some time had elapsed belore the administration of chloroform convulsions recurred after labour, and the patient did not make 80 good a recovery.

Fourthly, the danger to the child is increased by delay.

The cases I have given are too few to form a definite opinion, but I think the immediatertreatment with chloroform deserves

a further trial. Forced delivery is quite a different thing when a patient is fully underchloroform to what it is when there is not complete musculwr rélaxation.

\section{A PLEA FOR EVACUATION OF THE UTERUS IN UNRELIEVED CABES OF PUERPERAL FCLAMPSIA.}

Bx ERNEST BRICE, L R.C.P., L.R.C.S.EDIN., Landore, Glamorgan.

A PERUSAL of the discussion on the treatment of puerperal eclampsia at the recent meeting of the British Medical Association at Leicester impresses one point very strongly on the mind, and that is that the element of doubt and uncertainty has crept into the minds of our leading obstetricians as to what is the proper method of handling such cases, or even as to whether any treatment at all is called for. This is very unfortunate, for if there is any trying emergency in which a practitioner finds himself where prompt action seems called for. surely a case of this kind is one.

Now, is the impression one gathers from thls discussion a true representation of the fecling of the profession at large? I am aware that at the present day it is fashionable to doub everything; this is an age of doubt, and every one is tempted to question the wisdom of his fathers-and this is especially true in matters medical. It is true that we are bound to admit that, as science and art advance, new rays of light are shed upon what was once obscure, and we are bound often to sacrifice our household gods to new truths; yet I cannot help feeling that a grave danger exists of treating old loves too lightly ; of forgetting that old views, although not ideal ones, meant something, for they represented years of effcrt on the part of those who went before. True conservatism in medicine is a valuable principle. We must advance in thought and teaching; but we must think, not twice, but twenty times before we sweep away the wisdom of the growth of generations.

Applying these thoughts to the matter of the treatment of puerperal convulsions, I find that Meadows, in 1876, wrote :

"There is, indeed, little or no chance of the convulsion ceasing so long as the child remains in the uterus, with probably the head pressing upon a tender and irritable cervix, but no sooner is delivery accomplished than, as a general rule at least, the fit subsides; it may, of course, recur, but the patient's chances of recovery are decidedly better aiter delivery than before."

We may take this statement as representing the consensus of the opinion of his time, and the highest wisdom up to date : the acme of truth on the matter since the time when physicians began to consider it at all.

In 1891 , fifteen years later, Galabin wrote :

"No time should be lost in inducing labour, since nothing exercises so favourable an influence as the completion of delivery, both as regards the convulsions and the condition of the urine."

Here we have practically the same view expressed, namely, that hope only lay in the evacuation of the uterus; thus, after fifteen years' further research by obstetricians of no mean order, no better treatment can be suggested; the burden of treatment is still-evacuate the uterus.

Fifteen more years have pasged, and now doubt seems to be thrown upon the efficacy of this method. Why, it is difficult to say.

One can understand a new view of the pathology of a disease, or the discovery of a more effectual remedy altogether affecting our course of action, but of the pathology of puerperal eclampsia we have no more definite knowledge than we had twenty years ago, nor can we offer any better method of conducting a case. Yet the methods with which our forbears were successful are now being brought into question, and by some we are advised practically to leave things alone, "as we are as likely as not to do more harm than good by interference." One has the highest respect for those who express such views, and one feels that, coming from the source they do, they are bound to receive our most earnest consideration. Yet after all, it is hard to believe that the non-interference policy embodies the result of the experience of the whole body of the profession. It would be very helpful if the general practitioner could be induced to give his experience.

In thinking over cases of my own, I have in my mind six, in each of which relief followed the birth of the child. Unfortunately I have not an exact record of the cases, as notes were not taken at the time, but the details are substantially correct, and one fact is very plainly stamped upon my mind, and that is that the convulions were always relieved after the evacuation of the uterus. 